Disclosures M. Mokin: 2; C; Toshiba (Canon) Medical. C. Primiani: None. K. Piper: None. D. Fiorella: 1; C; reports grants, non-financial support, and other funding from Penumbra, Cerenovus, and Stryker; non-financial support and other funding from Genentech and Shape Memory Medical; and grants from Siemens. A. Rai: 6; C; reports other from Penumbra; and non-financial support from Microvention and Stryker. K. Orlov: None. D. Kislitsin: None. A. Gorbatykh: None. J. Mocco: 6; C; reports other from Penumbra, Cerebrotech, Rebound Therapeutics, TSP, Lazarus Effect, Medina, Pulsar Vascular, and Blockade. R. DeLeacy: 6; C; reports other and non-financial support from Penumbra; and non-financial support from Cerenovus and Siemens. J. Lee: None. A. Spiotta: 6; C; reports other and non-financial support from Penumbra, Pulsar Vascular, and Stryker and grants, other, and non-financial support from Microvention. R. Turner: 2; C; consultant for Penumbra, Medtronic, Microvention, Codman, Pulsar Vascular, Q'Apel, Rebound Medical, and Blockade Medical. I. Chaudry: 6; C; reports other, grants, and non-financial support from Penumbra and Pulsar Vascular; grants and nonfinancial support from Medtronic, Microvention, and Codman; non-financial support and other from Bloc. A. Turk: 6; C; reports other, grants, and non-financial support from Penumbra and Pulsar Vascular; grants and non-financial support from Codman, Microvention, and Medtronic; non-financial support and other from Bloc.

\section{0-024 COMPARISON BETWEEN OUTCOMES OF ANTERIOR AND POSTERIOR CEREBRAL CIRCULATION ANEURYSMS IN THE SMART REGISTRY COHORT}

E Almallouhi*, M Sattur, M Anadani, S Al kasab, A Spiotta. Medical University of South Carolina, Charleston, SC

\subsection{6/neurintsurg-2019-SNIS.24}

Background Endovascular treatment of cerebral aneurysms in the posterior circulation poses a challenge because of the higher rate of wide-necked aneurysms and presentation with rupture. In this study, we compare the baseline characteristics and outcomes of patients with posterior circulation aneurysms treated with novel second-generation coils in the SMART registry.

Methods SMART is a phase 4, multicenter, prospective, registry that enrolled patients with anterior and posterior circulation cerebral aneurysms (both ruptured and unruptured) who underwent endovascular treatment using Penumbra SMART ${ }^{\mathrm{TM}}$ Coils. Collected data included baseline clinical and angiographic characteristics at presentation. Primary and secondary end points were immediate angiographic occlusion, periprocedural complications, recanalization and retreatment rates and mortality at 1 -year follow up. We compared the above variables between anterior and posterior circulation aneurysms.

Results Of a total of 906 patients treated in the SMART registry, $173(19.1 \%)$ had posterior circulation aneurysms. In comparison to patients with anterior circulation aneurysms, patients with aneurysms in the posterior circulation were older (mean age 61.7 vs. 59.3 years, P 0.017 ) and more likely to present with rupture $(38.7 \%$ vs. $30.3 \%, \mathrm{P} 0.032)$. Posterior circulation aneurysms were more likely to be widenecked (defined as an aneurysm with a dome-to-neck ratio less than 2.0 and/or a neck length of $4 \mathrm{~mm}$ or more). No significant difference was found in the rate of successful embolization at initial procedure (Raymond Class I), serious device-related adverse effects within 24 hours, one-year recanalization and one-year retreatment between both groups (table 1).

\begin{tabular}{|c|c|c|c|}
\hline Baseline characteristics & $\begin{array}{l}\text { Posterior circulation } \\
\text { aneurysm patients } \\
(\mathrm{N}=173)\end{array}$ & $\begin{array}{l}\text { Anterior circulation } \\
\text { aneurysm patients } \\
(\mathrm{N}=733)\end{array}$ & $\begin{array}{l}P \text { - } \\
\text { value }\end{array}$ \\
\hline Age, Mean SD & 61.711 .6 & 59.312 .8 & 0.017 \\
\hline Female, $\%(n / N)$ & $78.6 \%(136 / 173)$ & $73.9 \%(542 / 733)$ & 0.203 \\
\hline $\begin{array}{l}\text { Ruptured aneurysm, \% } \\
(\mathrm{n} / \mathrm{N})\end{array}$ & $38.7 \%(67 / 173)$ & $30.3 \%(222 / 733)$ & 0.032 \\
\hline Aneurysm Size \% (n/N) & & & 0.285 \\
\hline Small (<11mm) & $86.1 \%(149 / 173)$ & $89.5 \%(656 / 733)$ & \\
\hline Large (11 to $25 \mathrm{~mm}$ ) & $13.3 \%(23 / 173)$ & $10.4 \%(76 / 733)$ & \\
\hline Giant $(>25 \mathrm{~mm})$ & $0.6 \%(1 / 173)$ & $0.1 \%(1 / 733)$ & \\
\hline Wide-Neck\% (n/N) & $72.2 \%(117 / 162)$ & $59.9 \%(439 / 733)$ & 0.002 \\
\hline $\begin{array}{l}\text { Stent-Assisted Coiling \% (n/ } \\
\text { N) }\end{array}$ & $38.2 \%(66 / 173)$ & $36.2 \%(265 / 733)$ & 0.624 \\
\hline $\begin{array}{l}\text { Balloon-Assisted Coiling \% } \\
\text { (n/N) }\end{array}$ & $16.8 \%(29 / 173)$ & $20.6 \%(151 / 733)$ & 0.255 \\
\hline Raymond Occlusion, Class I & & & \\
\hline $\begin{array}{l}\text { Immediate Post-Procedure\% } \\
(\mathrm{n} / \mathrm{N})\end{array}$ & $37.6 \%(65 / 172)$ & $41.1 \%(298 / 729)$ & 0.457 \\
\hline $\begin{array}{l}\text { One-Year Follow-Up \% } \\
\text { (n/N) }\end{array}$ & $55.1 \%(43 / 78)$ & $64.4 \%(250 / 388)$ & 0.121 \\
\hline $\begin{array}{l}\text { Recanalization at One-Year } \\
\text { Follow-Up \% (n/N) }\end{array}$ & $12.8 \%(10 / 78)$ & $14 \%(54 / 385)$ & 0.205 \\
\hline $\begin{array}{l}\text { Retreatment through } \\
\text { One-Year Follow-Up }\end{array}$ & $6.2 \%(5 / 81)$ & $7 \%(28 / 399)$ & 0.190 \\
\hline $\begin{array}{l}\text { Device-Related SAE within } \\
24 \text { Hours of Procedure \% } \\
(\mathrm{n} / \mathrm{N})\end{array}$ & $1.7 \%(3 / 173)$ & $3 \%(22 / 733)$ & 0.360 \\
\hline $\begin{array}{l}\text { Length of Hospital Stay, } \\
\text { Median [IQR] }\end{array}$ & $2[1-14]$ & $1[1-10]$ & 0.053 \\
\hline
\end{tabular}

Conclusions Despite being associated with higher rates of rupture and wide-necked geometry, patients with posterior circulation aneurysms experienced immediate and one-year angiographic occlusion rates that were not significantly different from anterior circulation aneurysms. Device-related periprocedural complications were also not significantly higher than the latter.

Disclosures E. Almallouhi: None. M. Sattur: None. M. Anadani: None. S. Al kasab: None. A. Spiotta: 2; C; Penumbra, Cerenovus, Minnetronix.

\section{0-025 SOUND MEASUREMENT IN PATIENT-SPECIFIC 3D PRINTED BENCH MODELS FOR VENOUS PULSATILE TINNITUS}

M Amans*, K Valluru, H Haraldsson, E Kao, J Leach, A Wright, M Ballweber, K Meisel, D Saloner. Radiology, UCSF, San Francisco, CA

\subsection{6/neurintsurg-2019-SNIS.25}

Introduction Pulsatile tinnitus (PT) can be caused by aberrant blood flow in large cerebral veins near the cochlea. In our previous works we evaluated flow patterns in patient's 

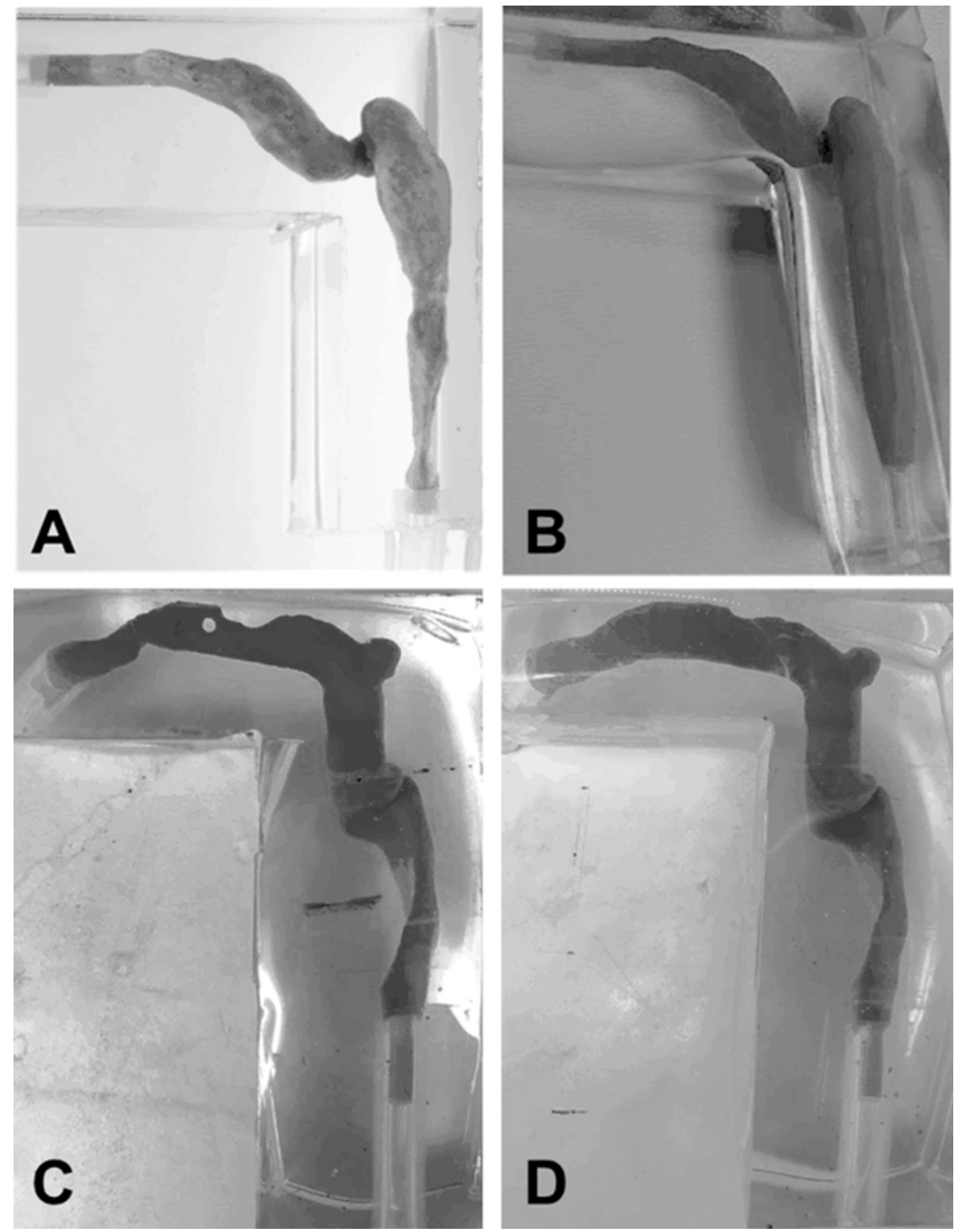

Abstract 0-025 Figure 1 A) 3D printed venous segment of patient one showing native cerebral venous sinus and jugular vein derived from the CE-MRA dataset, and B) The venous anatomy was reprinted after computationally modifying the internal jugular vein to remove the stenosis across C1 transverse process. 3D printed venous segment of patient two showing transverse sinus, sigmoid sinus, and internal jugular vein derived from CEMRA acquired: C) before lumbar puncture, and D) after lumbar puncture

cerebral venous sinuses to try and identify patterns of flow unique to symptomatic PT patients. However, the exact mechanism of sound production remains unclear. In order to further investigate the sound production mechanism, we created 3D-printed flow models based on patient-specific cerebral venous anatomies.

Methods We performed MR patients with venous etiology of PT recruited from the UCSF Pulsatile Tinnitus Clinic. A surface representation of the venous sinuses from the affected side was segmented from the CE-MRA dataset and modified by adding flow extensions at both ends to facilitate connections to a rotary pump. The lumenal anatomy was printed in wax that was embedded in a degassed solution of silicone encapsulant that was cured for 48 hours. The inner wax lumen was then melted out of the silicone by heating at $120^{\circ} \mathrm{C}$ creating a durable and rigid patient-specific flow model (figure 1). Models were attached to a pulsatile pump and sound recordings were made using a Bluetooth-compatible electronic stethoscope. Signals were processed and analyzed in MATLAB.

Results and discussion Four total flow models were created from two patients (figure 1). Patient one has a high-riding jugular bulb and stenosis of the internal jugular vein at the level of C1 (figure 1A). An additional modified flow model for this patient was created by computationally removing the stenosis (figure 1B). Other models are from a patient with idiopathic intracranial hypertension and a transverse sinus stenosis (figure 1C). After lowering her ICP via LP, her symptoms resolved, and an additional MRA was performed that was used to generate the post-LP (figure 1D). 
Conclusions We have developed patient-specific flow models for venous causes of PT that may be an appropriate in vitro surrogate for venous causes of PT.

Disclosures M. Amans: 1; C; NIH. 2; C; Stryker, Covidien. K. Valluru: None. H. Haraldsson: None. E. Kao: None. J. Leach: None. A. Wright: None. M. Ballweber: None. K. Meisel: None. D. Saloner: 1; C; NIH.

\section{0-026 PERSISTENT VENOUS PRESSURE GRADIENT AFTER VENOUS SINUS STENTING WITH RESOLUTION AFTER SECOND STENT PLACEMENT: A TECHNICAL NOTE}

H Dasenbrock*, A Beer-Furlan, M Chen, R Crowley. Neurosurgery, Rush University, Chicago, IL

\subsection{6/neurintsurg-2019-SNIS.26}

Object Although venous sinus stenting is an established treatment for medically refractory idiopathic intracranial hypertension (IIH), a subset of patients do not sustain improvement in symptoms post-procedurally. While many physicians perform post-stent manometry, this is not universal and there is no accepted practice regarding where pressures should be measured when pursued. In this report, two patients are described who had resolution of their pressure gradient across the stented region after venous sinus stenting, yet had a persistent physiologic venous pressure gradient elsewhere. After placement of a second stent during the same procedure, subsequent resolution of the gradient was achieved.

Methods This retrospective chart review evaluated patients at a single institution who underwent venous sinus stenting and had follow-up of at least one-year.

Results Two patients (41F and $42 \mathrm{~F}$ ) with medically refractory IIH underwent angiography with venous manometry. In the first patient, stenosis was observed at the transverse-sigmoid sinus junctions bilaterally. The maximum venous pressure was $40 \mathrm{mmHg}$, and a gradient of $30 \mathrm{mmHg}$ was present across the right transverse-sigmoid junction, where a unilateral venous sinus stent (Zilver, Cook Medical, Bloomington IN) was placed. Post-stent pressure measurements showed there was no longer a gradient from the transverse to sigmoid sinus. However, manometry in the superior sagittal sinus revealed that a significant pressure gradient persisted, which resolved after an additional stent was placed in the contralateral transverse sinus. The second patient had unilateral venous stenosis and a maximum pressure of $50 \mathrm{mmHg}$. Following placement of a right transverse-sigmoid sinus stent, the gradient was eliminated and pressures normalized in the transverse sinus. Upon measuring pressures in the superior sagittal sinus, the maximum venous pressures had only decreased to $30 \mathrm{mmHg}$, with a persistent gradient of $20 \mathrm{mmHg}$ between the superior sagittal sinus and the right transverse sinus. An additional stent was placed across this location, and the pressure gradient resolved completely. Both patients had improvement in their symptoms, which has been sustained at one-year follow-up.

Conclusions In some patients with IIH and venous sinus stenosis, placement of a single stent may eliminate the pressure gradient across the transverse-sigmoid sinus junction, yet may not sufficiently normalize pressures throughout the venous system. This may account for some patients who 'fail' venous sinus stenting. Additionally, manometry on both sides of the stent alone would not have detected a persistent pressure gradient, which was only detected with measurement in the superior sagittal sinus. We therefore advocate for measuring pressures in the superior sagittal sinus following stent placement, regardless of the location of the stent, which may detect the presence of a second clinically relevant stenosis, and an additional stent may be needed.

Disclosures H. Dasenbrock: None. A. Beer-Furlan: None. M. Chen: 2; C; Genentech, Pneumbra, Stryker, Medtronic. R. Crowley: None.

\section{0-027 MR DERIVED TTP PERFUSION MAPS ARE PREDICTIVE OF THE RATE OF STROKE EVOLUTION}

R King*, O Brooks, M Gounis, M Shazeeb. Radiology, University of Massachusetts, Worcester, MA

\subsection{6/neurintsurg-2019-SNIS.27}

Introduction Thrombectomy has rates of successful recanalization of nearly $85 \%{ }^{1}$; however, many patients may not be eligible or not have a successful outcome following recanalization if the infarct evolves prior to arriving to the angiosuite. Neuroprotection that 'freezes' the penumbra to allow a longer window to receive treatment has become the next critical topic in stroke care $^{2}$. The canine temporary large vessel occlusion model has been recently developed to allow for assessment of neuroprotectants. A limitation of this model is the rate of stroke evolution tends towards one of two pathways: fast evolution, where more than half the total infarct volume is present within 90 minutes; and slow evolution, where less than half the final volume occurs within 90 minutes. Here we present a novel analysis of MR based Timeto-Peak (TTP) maps, derived from perfusion-weighted MRI (PW-MRI), to classify the canine stroke evolution based on the first PW-MRI.

Methods Fourteen dogs were retrospectively analyzed for patterns of stroke evolution. An autologous clot was injected into the MCA in order to cause an occlusion. Once the occlusion was confirmed, the animal was moved to the MRI scanner. MRI protocol included Time of Flight (ToF), Diffusion weighted (DWI) and perfusion weighted (PWI) imaging. For PWI $0.2 \mathrm{mmol} / \mathrm{kg}$ gadopentetate dimeglumine was injected IV. To calculate the true volume of stroke, ADC maps were generated from the DWI images. The TTP maps were normalized relative to the unaffected contralateral half of the brain. The TTP map was registered to the DWI images to only account for voxels within the stroke volume. Finally, all voxels of the TTP map within the ADC lesion were binned into one of four categories: slight delay (4-8s), moderate delay (9-13s), long delay (14-18s) and extended delay ( $\geq 19 \mathrm{~s})$.

Results Using the DWI evolution as the ground truth, the canines were categorized into two groups of fast and slow evolvers (figure 1A). The binned TTP maps in the slight delay and extended delay groups also accurately predicted the evolution pathway in classifying all the canines in this study (figure $1 \mathrm{~B}$, $\mathrm{p}<0.01)$.

Conclusions The ability to predict the rate of stoke evolution, at the point of first MRI, will allow for a better 